\title{
Antimicrobial resistance: A continuing Canadian tale
}

\author{
LE Nicolle MD FRCPC, Editor-in-Chief
}

In 1997, Health Canada and the Canadian Infectious Diseases Society coordinated the Canadian Consensus Conference on Antimicrobial Resistance. The Action Plan developed at this meeting was a series of recommendations for a Canadian initiative to address the serious and expanding problem of resistance (1). Other national and international documents have since repeated this theme, and national strategies have been developed for other countries, including Britain (2) and the United States (3). The World Health Organization proposed a Global Strategy for addressing antimicrobial resistance in 2001 (4). Since 1997, flouroquinolone-resistant Streptococcus pneumoniae, flouroquinolone-resistant Salmonella typhi, trimethoprim/ sulfamethoxazole-resistant community Escherichia coli, flouroquinolone-resistant community E coli, extended spectrum beta-lactamase-producing $E$ coli, vancomycinintermediate Staphylococcus aureus, and now vancomycinresistant $S$ aureus have become issues of clinical and public health concern. In Canadian hospitals, methicillinresistant $S$ aureus (MRSA) continues to increase (5) and Clostridium difficile with high-level resistance to clindamycin and flouroquinolones is widespread. Although Canada was among the first countries to propose a national agenda to contain antimicrobial resistance, five years later the antimicrobial resistance initiative appears to have languished. Antimicrobial resistance evolves - is Canada standing still?

One recommendation of the 1997 conference was the formation of a coordinating committee to provide leadership and to take responsibility for forwarding the Canadian Action Plan. The Canadian Committee on Antimicrobial Resistance (CCAR) was formed within a year, and has spearheaded the antimicrobial resistance containment agenda through an advocacy and promotion role. A CCAR summary of Canadian activities relevant to resistance is published in The Canadian Journal of Infectious Diseases twice yearly. CCAR has been restricted, however, in its pursuit of the resistance agenda by limited resources, with support primarily through short term funding from the federal government. In early October, 2002, CCAR organized a National Canadian Policy Conference on Antimicrobial Resistance. The goal of this conference was to review the current status of antimicrobial resistance in Canada in the context of the global experience, and to revitalize and refocus the national action plan to limit antimicrobial resistance.

One theme of the conference was frustration. Frustration at the apparent failure of the antimicrobial resistance issue to capture government attention or the public policy agenda. Frustration at the lack of progress for infrastructure development for data collection systems to measure and monitor antimicrobial use and resistance. Frustration at the rising rates of MRSA and other key organisms in health care institutions despite intensive infection control measures. Frustration at the failure to secure dedicated resources for effective antimicrobial containment programs, including a lack of consistent funding for CCAR. And, certainly, frustration at the high talk-to-action ratio for this topic.

There was also a shining light - the draft report, "Uses of antimicrobials in food animals in Canada: impact on resistance in human health" by the Advisory Committee on Animal Uses of Antimicrobials and Impact on Resistance and Human Health (7). The report, prepared for the Veterinary Drugs Directorate, summarized the controversial, polarized and complex issues of antimicrobial use for agriculture in Canada. The recommendations of the report, including greater controls on prescribing, limitations of antimicrobial use for growth promotion, and development of a national surveillance system for antimicrobial resist-

Health Sciences Centre, Department of Internal Medicine, Winnipeg, Manitoba

Correspondence: Dr LE Nicolle, Health Sciences Centre, Department of Internal Medicine, CG443-820 Sherbrook Street, Winnipeg,

Manitoba R3A 1R9. Telephone 204-787-7029, fax 204-787-4826, e-mail nicolle@cc.umanitoba.ca 
ance in food animal production are essential to addressing antimicrobial resistance nationally. The full implementation of all recommendations is pending, but progress toward a national surveillance program is apparent, with resources committed by the federal government to support development. The initial impetus for this funding was not concern for health or resistance, but rather that current Canadian practices in the use of antimicrobials in food animals jeopardize potential international trade opportunities.

The conference goal was to identify a limited number of key areas where efforts should be focused to most effectively move the National Action Plan forward. After two days of intense discussion, the five issues proposed were monitoring antibiotic use, developing a multidisciplinary national education program for the appropriate use of antibiotics, ensuring effective infection control and prevention methods in all sectors, developing an integrated surveillance plan with external quality assurance for human and animal antimicrobial resistance, and implementing the animal recommendations contained in the Veterinary Drugs Directorate Health Canada Report. These are the same themes recurring in all strategies to address resistance - surveillance, education, infection prevention and animal husbandry. Apart from the animal husbandry initiative, are we likely to have more success in implementing the other agenda priorities now than we did in 1997 ?

Monitoring antimicrobial use seems to be the most straightforward objective. Provinces currently collect outpatient antimicrobial prescribing information within pharmacare programs, and some already have a relevant analytical framework. Surveillance and reporting back to prescribers, by themselves, may influence use. Standardized descriptors such as defined daily doses need to be applied, and the relative use of antimicrobials should be compared among physician groups within a province, and usage should be compared across provinces. Some programs are already in existence in New Brunswick and Saskatchewan. The example of Denmark was before us throughout the meeting. Canada is larger, with a more diverse population and a more complex health care delivery system than Denmark, but opportunities for rapid implementation already exist in some provinces in Canada. While antimicrobial resistance may not be a priority for the provincial government, antimicrobial use analysis will benefit provincial drug plans beyond resistance considerations. It is generally accepted that a critical and appropriate program for the evaluation of antimicrobial use is likely to lead to decreased use, given consistent evidence of substantial antimicrobial overuse throughout the health care system.

There are also several small-scale, Canadian, regional education programs to modify antimicrobial use in which outcomes of decreased use have been reported (8). Whether such programs are applicable at a larger scale, or will have sustained results, are open questions. However, the limitation of antimicrobial use for selected outpatient infections is a message that the public appears willing to hear. But there are pitfalls here, too. Decreased outpatient prescribing in the United States has been paralleled by a trend to less use of older, first-line agents, and greater use of more recently introduced broad-spectrum antimicrobials. This reinforces the need for critical monitoring and analysis of antimicrobial use trends.

The implementation of effective infection prevention methods is also controversial. Vaccine programs are everexpanding and, at least for childhood illnesses, may decrease overall antimicrobial use as well as infection with antimicrobial-resistant organisms such as Haemophilus influenzae and $S$ pneumoniae. Hygiene messages are also widespread, but have, in part, been perverted to the widespread promotion of household disinfectant use, which may actually promote resistance. Within acute care facilities, infection control programs have often been hampered by the antimicrobial resistance agenda. Limited institutional resources in infection control are applied to colonization prevention rather than to infection prevention, and program components of proven benefit in infection reduction may languish. Achieving appropriate resources for both infection control and antimicrobial resistance containment is essential.

Developing a surveillance program for antimicrobialresistant organisms is complex, at least partially because the goals for such a program have not been clearly articulated. How do we select organisms of interest? How does surveillance evolve to remain timely and relevant as antimicrobial resistance and use evolves? How can surveillance bridge the spectrum of health care delivery from community to critical care? Should both colonization and infection be measured? How are morbidity and potential mortality assessed? A limited, focused surveillance for selected organisms of high interest is likely the most effective way to start. This approach has proved to be successful with the Canadian Nosocomial Infection Surveillance Program, which has measured trends and impacts of MRSA (5) and vancomycin-resistant enterococci (9) in Canada. The development of an effective surveillance program to serve public health policy remains a great challenge.

Antimicrobial-resistant organisms will be an integral part of our environment as long as antimicrobial agents are used. We need to live effectively with all of the outcomes of antimicrobial use, both good and bad. The recent CCAR conference reminds us that the principle themes of antimicrobial resistance containment do not change. In Canada, despite a relatively early start, limited progress has been made in addressing this problem. The status quo in antimicrobial use has a clear future - ever increasing antimicrobial use, antimicrobial resistance and costs. An alternate path is clear and has been repeatedly articulated, including at the recent CCAR conference. Antimicrobial resistance containment is a clinical, public health and economic imperative. This country needs to move aggressively beyond the Action Plan, to the action. 


\section{REFERENCES}

1. Health Canada. Controlling antimicrobial resistance. An integrated action plan for Canadians. Can Comm Dis Rep 1997;23(Suppl7):1-32.

2. Standing Medical Advisory Committee, Sub-group on Antimicrobial Resistance: Main Report. The path of least resistance. London: Department of Health, 1998. <http://www.doh.gov.uk/arbstrat.htm>.

3. CDC, FDA, NIH: A Public Health Action Plan to Combat Antimicrobial Resistance. <www.cdc.gov/drugresistance/actionplan/ index.htm>. Version current November 22, 2002.

4. WHO Global Strategy for Containment of Antimicrobial Resistance WHO/CDS/CSR/DRS 2001-2. <http://www.who.int/ emc-documents/antimicrobial_resistance/docs/EGlobal_Strat.pdf> Version current November 22, 2002.

5. Simor AE, Ofner-Agostini M, Bryce E, et al, for Canadian Nosocomial Infection Surveillance Program, Health Canada. The evolution of methicillin-resistant Staphylococcus aureus in Canadian hospitals: 5 years of national surveillance. CMAJ 2001;165:21-32.

6. Conly J, Canadian Committee on Antibiotic Resistance. Antimicrobial resistance in Canada: Update on activities of the Canadian Committee on Antibiotic Resistance. Can J Infect Dis 2002;13:236-8.

7. Health Canada, Health Products and Food Branch. Uses of antimicrobials in food animals in Canada: Impact on resistance and human health. <http://www.hc-sc.gc.ca/vetdrugs-medsvet>. Version current at November 27, 2002.

8. Conly J. Antimicrobial resistance in Canada. CMAJ 2002;167:885-91.

9. Ofner-Agostini ME, Conly J, Paton S, et al. Vancomycin-resistant enterococci in Canada - results of the Canadian Nosocomial Infection Surveillance Program 1996: VRE point prevalence surveillance project. Can J Infect Dis 1997;8:73-8. 


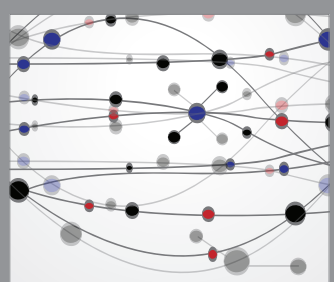

The Scientific World Journal
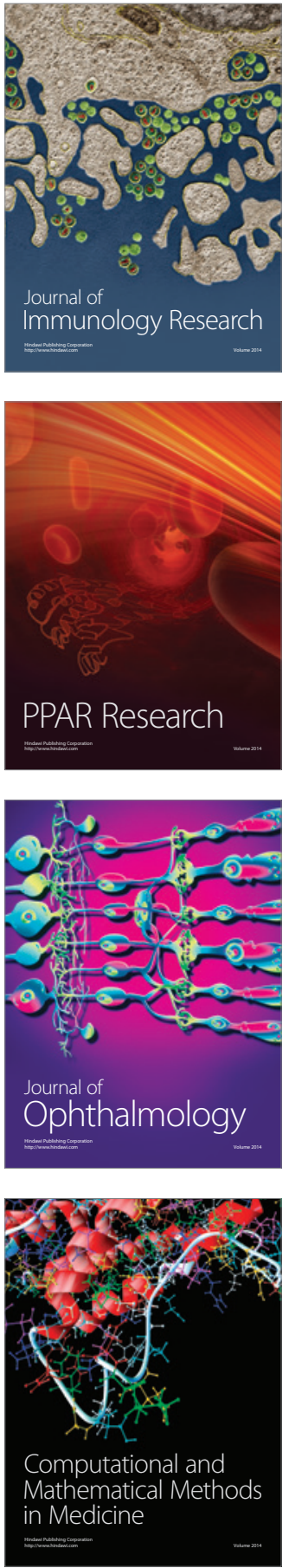

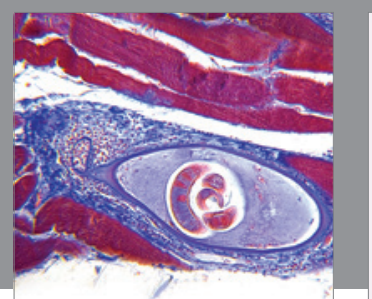

Gastroenterology Research and Practice

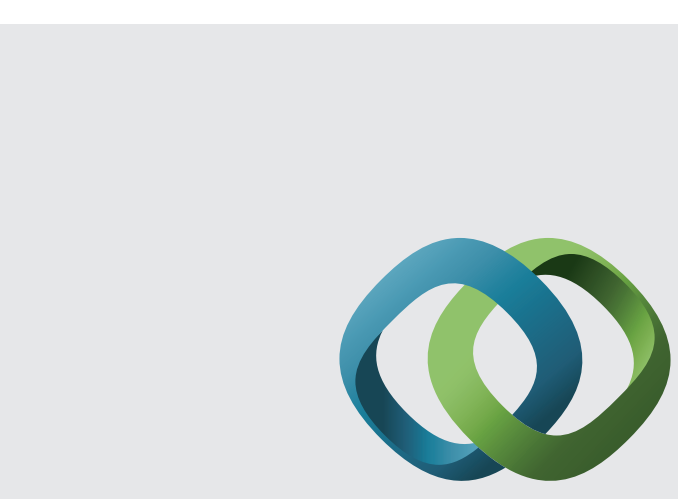

\section{Hindawi}

Submit your manuscripts at

http://www.hindawi.com
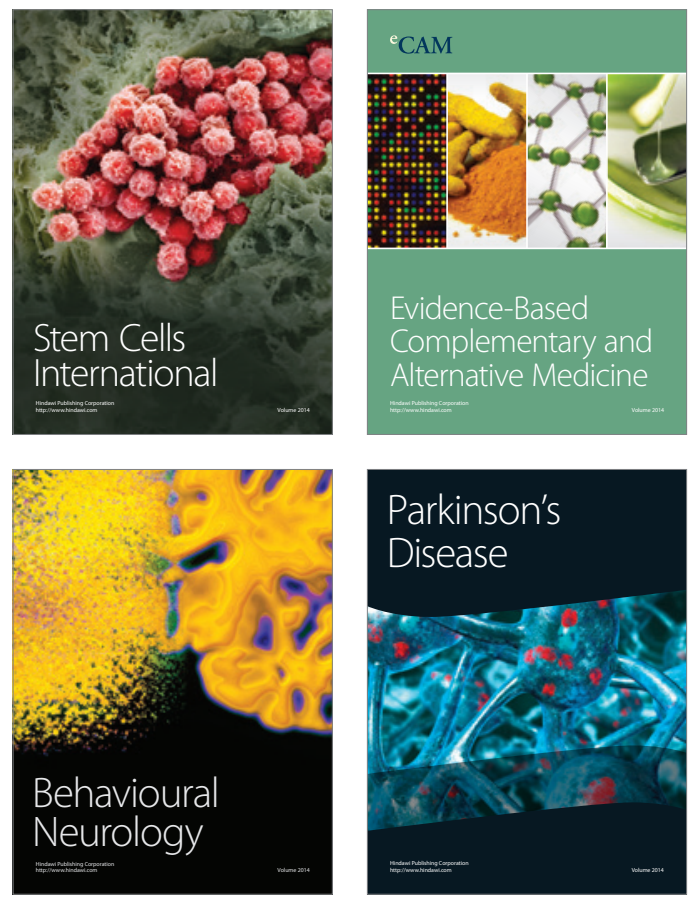
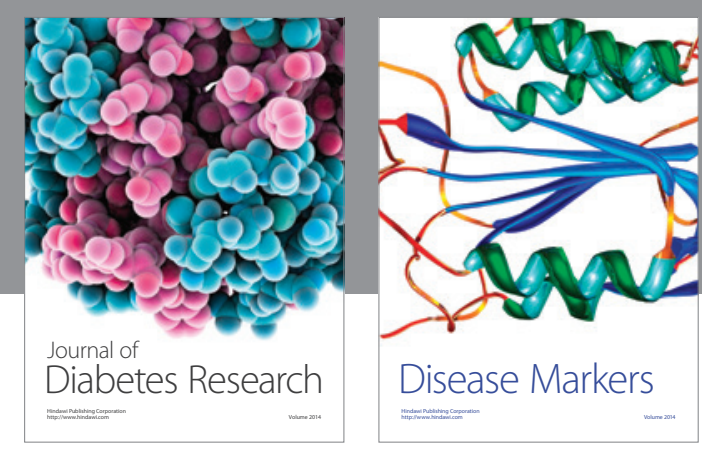

Disease Markers
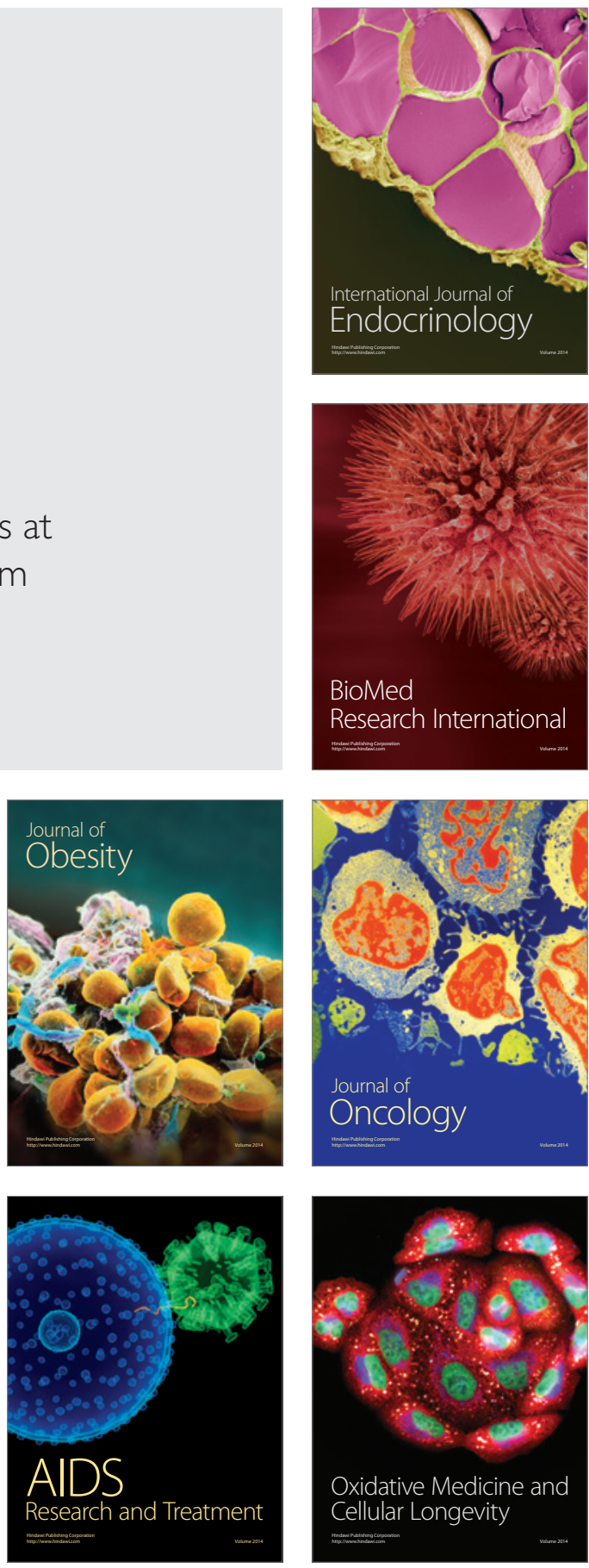